CUBO A Mathematical Journal

Vol.12, $N^{\underline{Q}} 01$, (161-174). March 2010

\title{
Convergence Conditions for the Secant Method
}

\author{
IOANNIS K. ARgYros \\ Department of Mathematics Sciences, \\ Lawton, OK 73505, USA \\ email: iargyros@cameron.edu \\ AND \\ SAÏD HilOUT \\ Poitiers university, \\ Laboratoire de Mathématiques et Applications, \\ 86962 Futuroscope Chasseneuil Cedex, France \\ email: said.hilout@math.univ-poitiers.fr
}

\begin{abstract}
We provide new sufficient convergence conditions for the convergence of the Secant method to a locally unique solution of a nonlinear equation in a Banach space. Our new idea uses recurrent functions, Lipschitz-type and center-Lipschitz-type instead of just Lipschitz-type conditions on the divided difference of the operator involved. It turns out that this way our error bounds are more precise than earlier ones and under our convergence hypotheses we can cover cases where earlier conditions are violated. Numerical examples are also provided in this study.
\end{abstract}

\section{RESUMEN}

Son dadas nuevas condiciones suficientes para la convergencia del método de la secante para una solución localmente única de una ecuación no lineal en un espacio de Banach. Estas ideas nuevas usan funciones recurrentes, tipo-Lipschitz y tipo centro-Lipschitz sobre la diferencia dividida de los operadores envolvidos. Resulta que esta manera las cotas de errores son mas 
precisas que las anteriores y bajo nuestras hipótesis de convergencia nosotros podemos cubrir casos donde las condiciones previas eran violadas. Ejemplos numéricos son dados en este estudio.

Key words and phrases: Secant method, Banach space, majorizing sequence, divided difference, Fréchet-derivative.

Math. Subj. Class.: 65H10, 65B05, 65G99, 65N30, 47H17, 49M15.

\section{Introduction}

In this study we are concerned with the problem of approximating a locally unique solution $x^{\star}$ of equation

$$
F(x)=0,
$$

where $F$ is a Fréchet-differentiable operator defined on a convex subset $\mathcal{D}$ of a Banach space $\mathcal{X}$ with values in a Banach space $\mathcal{Y}$.

A large number of problems in applied mathematics and also in engineering are solved by finding the solutions of certain equations. For example, dynamic systems are mathematically modeled by difference or differential equations, and their solutions usually represent the states of the systems. For the sake of simplicity, assume that a time-invariant system is driven by the equation $\dot{x}=T(x)$, for some suitable operator $T$, where $x$ is the state. Then the equilibrium states are determined by solving equation (1.1). Similar equations are used in the case of discrete systems. The unknowns of engineering equations can be functions (difference, differential, and integral equations), vectors (systems of linear or nonlinear algebraic equations), or real or complex numbers (single algebraic equations with single unknowns). Except in special cases, the most commonly used solution methods are iterative-when starting from one or several initial approximations a sequence is constructed that converges to a solution of the equation. Iteration methods are also applied for solving optimization problems. In such cases, the iteration sequences converge to an optimal solution of the problem at hand. Since all of these methods have the same recursive structure, they can be introduced and discussed in a general framework.

We consider the Secant method in the form

$$
x_{n+1}=x_{n}-\delta F\left(x_{n-1}, x_{n}\right)^{-1} F\left(x_{n}\right) \quad(n \geq 0), \quad\left(x_{-1}, x_{0} \in \mathcal{D}\right)
$$

where $\delta F(x, y) \in \mathcal{L}(\mathcal{X}, \mathcal{Y})(x, y \in \mathcal{D})$ is a consistent approximation of the Fréchet-derivative of $F$ [5], [13]. Bosarge and Falb [7], Dennis [9], Potra [16], Argyros [1]-[5], Gutiérrez [10] and others [11], [15], [18], have provided sufficient convergence conditions for the Secant method based on Lipschitz-type conditions on $\delta F$. 
The sufficient convergence condition for the Secant method used in most references is given

$$
\ell c+2 \sqrt{\ell \eta} \leq 1
$$

where, $\ell, c, \eta$ are non-negative parameters to be precised later. This hypothesis is easily violated. Indeed, let $\ell=1, \eta=.18$, and $c=.185$. Then, (1.3) does not holds, since $\ell c+2 \sqrt{\ell \eta}=$ 1.033528137. Hence, there is not guarantee that an equation using the information $(\ell, c, \eta)$ has a solution that can be found using Secant method (1.2). In this study we are motived by optimization considerations, and the above observation.

Here, using recurrent functions, Lipschitz-type and center-Lipschitz-type conditions, we provide a semilocal convergence analysis for (1.2). It turns out that our error bounds are more precise and our convergence conditions hold in cases where the corresponding hypotheses mentioned in earlier references mentioned above are violated. Newton's method is also examined as a special case. Numerical examples are also provided in this study.

\section{Semilocal Convergence Analysis of the Secant Method}

We need the following result on majorizing sequences for the Secant method (1.2).

Lemma 2.1. Let $\ell_{0}>0, \ell>0, c>0$, and $\eta \in[0, c]$ be given parameters.

Assume:

$$
\ell_{0}(c+\eta)<1
$$

Set

$$
\delta_{0}=\frac{\ell(c+\eta)}{c\left(1-\ell_{0}(c+\eta)\right)}
$$

Moreover, assume: there exists $\delta \in\left[\max \left\{\ell, \delta_{0}\right\}, \frac{1}{c}\right)$, such that estimate

$$
\ell q+\ell_{0}\left(\frac{2 q^{1 / \sqrt{5}}}{1-q^{(p-1) / \sqrt{5}}}+q^{2}+q\right)+\ell-\delta \leq 0
$$

is satisfied for $q=\delta c$, and $p=\frac{1+\sqrt{5}}{2}$.

Then, scalar sequence $\left\{t_{n}\right\}(n \geq-1)$ given by

$$
t_{-1}=0, t_{0}=c, t_{1}=c+\eta, t_{n+2}=t_{n+1}+\frac{\ell\left(t_{n+1}-t_{n-1}\right)\left(t_{n+1}-t_{n}\right)}{1-\ell_{0}\left(t_{n+1}-t_{0}+t_{n}\right)}
$$


is non-decreasing, bounded above by

$$
t^{\star \star}=\frac{c}{q} \sum_{n=1}^{\infty} q^{u_{n}}
$$

and converges to some $t^{\star} \in\left[0, t^{\star \star}\right]$, where $\left\{u_{n}\right\}$ is the Fibonacci's sequence: $u_{-1}=u_{0}=1, \quad u_{n+1}=$ $u_{n}+u_{n-1}, n \geq 1$.

Moreover, the following a priori estimates hold:

$$
0 \leq t^{\star}-t_{n} \leq \frac{c\left(q^{1 / \sqrt{5}}\right)^{p^{n}}}{q\left(1-q^{p^{n}(p-1) / \sqrt{5}}\right)} \quad(n \geq 0) .
$$

Proof. We shall show using induction on $k$ :

$$
\begin{gathered}
\frac{\ell\left(t_{k+1}-t_{k-1}\right)}{1-\ell_{0}\left(t_{k+1}-t_{0}+t_{k}\right)} \leq \delta\left(t_{k}-t_{k-1}\right), \\
0 \leq t_{k+1}-t_{k} \leq t_{k}-t_{k-1},
\end{gathered}
$$

and

$$
t_{k+1}-t_{k} \leq q^{u_{k}-1} c
$$

Note that if (2.10) holds, then by (2.7), we have:

$$
t_{k+2}-t_{k+1}=\frac{\ell\left(t_{k+1}-t_{k-1}\right)\left(t_{k+1}-t_{k}\right)}{1-\ell_{0}\left(t_{k+1}-t_{0}+t_{k}\right)} \leq \delta\left(t_{k}-t_{k-1}\right)\left(t_{k+1}-t_{k}\right) .
$$

Estimate (2.10) can also be written as:

$$
t_{k+1}-t_{k} \leq \alpha_{k}\left(t_{k}-t_{k-1}\right)
$$

where,

$$
\alpha_{k}=\frac{1}{\ell}\left(\delta\left(1-\ell_{0}\left(t_{k+1}-t_{0}+t_{k}\right)\right)-\ell\right) \text {. }
$$

For $k=0,(2.14)$ becomes

$$
\eta \leq \frac{1}{\ell}\left(\delta\left(1-\ell_{0}(c+\eta)\right)-\ell\right) c, \quad \text { or } \quad \delta \geq \delta_{0},
$$

which is true by the choice of $\delta$. Estimates (2.11) and (2.12) also hold for $k=0$ by the initial conditions. 
Assume that (2.10) (i.e., (2.13)), (2.11), and (2.12) hold for all $k \leq n$.

By the induction hypotheses we have

$$
0 \leq t_{k+2}-t_{k+1} \leq \delta q^{u_{k-1}-1} c\left(t_{k+1}-t_{k}\right)=q^{u_{k-1}}\left(t_{k+1}-t_{k}\right)<t_{k+1}-t_{k} .
$$

That is (2.11) holds for $n=k+1$. On the other hand:

$$
0 \leq t_{k+2}-t_{k+1} \leq q^{u_{k-1}}\left(t_{k+1}-t_{k}\right) \leq q^{u_{k-1}} q^{u_{k}} c=q^{u_{k+1}} c
$$

which shows (2.12) for $n=k+1$.

We also have the estimate

$$
\begin{aligned}
0 \leq t_{k+2}-t_{0} & \leq\left(t_{1}-t_{0}\right)+\left(t_{2}-t_{1}\right)+\cdots+\left(t_{k+2}-t_{k+1}\right) \\
& \leq \frac{c}{q}\left(q^{u_{0}}+q^{u_{1}}+\cdots+q^{u_{k}}\right)<t^{\star} .
\end{aligned}
$$

Clearly, we have:

$$
u_{k}=\frac{1}{\sqrt{5}}\left[\left(\frac{1+\sqrt{5}}{2}\right)^{k+1}-\left(\frac{1-\sqrt{5}}{2}\right)^{k+1}\right] \geq \frac{1}{\sqrt{5}}\left(\frac{1+\sqrt{5}}{2}\right)^{k}=\frac{p^{k}}{\sqrt{5}},
$$

So, for any $k \geq 0, m \geq 0$, we get in turn:

$$
\begin{aligned}
0 \leq t_{k+m}-t_{k} & \leq\left(t_{k}-t_{k+1}\right)+\left(t_{k+1}-t_{k+2}\right)+\cdots+\left(t_{k+m}-t_{k+m-1}\right) \\
& \leq \frac{c}{q}\left(q^{u_{k}}+q^{u_{k+1}}+\cdots+q^{u_{k+m-1}}\right) \\
& \leq \frac{c}{q}\left(q^{p^{k} / \sqrt{5}}+q^{p^{k+1} / \sqrt{5}}+\cdots+q^{p^{k+m-1} / \sqrt{5}}\right) .
\end{aligned}
$$

Using Bernoulli's inequality, we obtain:

$$
\begin{aligned}
t_{k+m}-t_{k} & \leq \frac{c}{q} q^{p^{k} / \sqrt{5}}\left(1+q^{\left(p^{k+1}-p^{k}\right) / \sqrt{5}}+q^{\left(p^{k+2}-p^{k}\right) / \sqrt{5}} \cdots+q^{\left(p^{k+m-1}-p^{k}\right) / \sqrt{5}}\right) \\
& =\frac{c}{q} q^{p^{k} / \sqrt{5}}\left(1+q^{p^{k}(p-1) / \sqrt{5}}+q^{p^{k}\left(p^{2}-1\right) / \sqrt{5}} \cdots+q^{p^{k}\left(p^{m-1}-1\right) / \sqrt{5}}\right) \\
& \leq \frac{c}{q} q^{p^{k} / \sqrt{5}}\left(1+q^{p^{k}(p-1) / \sqrt{5}}+q^{p^{k}(1+2(p-1)-1) / \sqrt{5}} \cdots+q^{p^{k}\left(p^{m-1}-1\right) / \sqrt{5}}\right) \\
& =\frac{c}{q} q^{p^{k} / \sqrt{5}}\left(1+q^{p^{k}(p-1) / \sqrt{5}}+\left(q^{p^{k}(p-1) / \sqrt{5}}\right)^{2} \cdots+\left(q^{p^{k}(p-1) / \sqrt{5}}\right)^{m-1}\right) \\
& =\frac{c}{q} q^{p^{k} / \sqrt{5}} \frac{1-q^{p^{k}(p-1) m / \sqrt{5}}}{1-q^{p^{k}(p-1) / \sqrt{5}} .}
\end{aligned}
$$

In particular, from (2.18) we have: 


$$
t_{k+m} \leq \frac{c}{q}\left(q^{u_{0}}+\cdots+q^{u_{k+m-1}}\right)+c
$$

In order for us to show (2.10), it suffices:

$$
\delta\left(t_{k}-t_{k-1}\right) \leq \alpha_{k+1}
$$

or

or

$$
\delta\left(t_{k}-t_{k-1}\right) \leq \frac{1}{\ell}\left(\delta\left(1-\ell_{0}\left(t_{k+2}-t_{0}+t_{k+1}\right)\right)-\ell\right)
$$

$$
\ell \delta q^{u_{k-1}-1}+\delta \ell_{0}\left(t_{k+2}+t_{k+1}-t_{0}\right) \leq \delta-\ell
$$

or

or

or

$$
\begin{aligned}
& \ell \delta q^{u_{k-1}-1} c+\delta \ell_{0}\left(\frac{c}{q}\left(q^{u_{0}}+q^{u_{1}}+\cdots+q^{u_{k+1}}\right)+c+\right. \\
& \left.\frac{c}{q}\left(q^{u_{0}}+q^{u_{1}}+\cdots+q^{u_{k}}\right)+c-c\right) \leq \delta-\ell
\end{aligned}
$$

$$
\ell \delta q^{u_{k-1}-1} c+\ell_{0}\left(2\left(q^{u_{0}}+q^{u_{1}}+\cdots+q^{u_{k}}\right)+q^{u_{k+1}}+\delta c\right) \leq \delta-\ell
$$

$$
\ell q^{u_{k-1}}+\ell_{0}\left(2\left(q^{u_{0}}+q^{u_{1}}+\cdots+q^{u_{k}}\right)+q^{u_{k+1}}+q\right) \leq \delta-\ell
$$

or

$$
\ell q+\ell_{0}\left(\frac{2 q^{1 / \sqrt{5}}}{1-q^{(p-1) / \sqrt{5}}}+q^{2}+q\right)+\ell-\delta \leq 0
$$

which is true by the choice of $\delta$ and $q$ given by (2.6).

The induction for (2.10)-(2.12) is now complete.

It follows from $(2.21)$ that scalar sequence $\left\{t_{n}\right\}$ is Cauchy in the complete space $\mathbb{R}$, and as such it converges to some $t^{\star} \in\left[0, t^{\star \star}\right]$. By letting $m \longrightarrow 0$ in $(2.21)$, we obtain $(2.9)$.

That completes the proof of Lemma 2.1.

We shall also provide another result where condition (2.6) is dropped from the hypotheses of Lemma 2.1:

Lemma 2.2. Let $\ell_{0}>0, \ell>0, c>0$, and $\eta \in(0, c]$ be given parameters.

Assume:

$$
\ell_{0}(c+\eta)<1
$$


there exists

$$
\delta \in\left(\max \left\{\ell+2 \ell_{0}, \delta_{0}, \delta_{1}\right\}, \min \left\{\frac{1}{c}, \delta_{\infty}\right\}\right)
$$

and,

$$
v_{1} \geq \delta_{1},
$$

where, $\delta_{1}=\frac{q_{1}}{c}$, is such that $q_{1}$ is the unique zero in $(0,1)$ of equation

$$
Q(t)=\ell_{0} t^{4}+\ell_{0} t^{2}+\ell t-\ell=0,
$$

$\delta_{\infty}=\frac{q_{\infty}}{c}$, is such that $q_{\infty}$ is the unique positive zero of equation

$$
f_{\infty}(t)=\ell_{0}\left(\frac{2 t^{1 / \sqrt{5}}}{1-t^{(p-1) / \sqrt{5}}}+t\right)+\ell-\delta=0,
$$

and

$v_{1}$ is the unique positive zero of equation

$$
f_{0}(t)=\ell_{0} t^{2}+\left(3 \ell_{0}+\ell\right) t+2 \ell_{0}+\ell-\delta=0 ;
$$

and

$$
\ell_{0} t^{2 u_{k}}+\ell_{0} t^{u_{k}}+\ell t^{u_{k-2}}-\ell \geq 0 \quad(k \geq 1) \quad \text { for } \quad t \geq \delta_{1} .
$$

Then, the conclusions of Lemma 2.1 hold true.

\section{Proof.}

We follow the proof of Lemma 2.1 until estimate (2.23). Then, let us define functions $f_{k}, g_{k}$ by

$$
f_{k}(t)=\ell t^{u_{k-1}}+\ell_{0}\left(2\left(1+t^{u_{1}}+\cdots+t^{u_{k}}\right)+t^{u_{k+1}}+t\right)+\ell-\delta,
$$

and

$$
g_{k}(t)=\bar{g}_{k}(t) t^{u_{k-1}}
$$

where,

$$
\bar{g}_{k}(t)=\ell_{0} t^{2 u_{k}}+\ell_{0} t^{u_{k}}+\ell t^{u_{k-2}}-\ell .
$$


We need to find the relationship between two consecutive functions $f_{k}$ :

$$
\begin{aligned}
f_{k+1}(t)= & \ell t^{u_{k}}+\ell_{0}\left(2\left(t^{0}+t^{u_{1}}+\cdots+t^{u_{k+1}}\right)+t^{u_{k+2}}+t\right)+\ell-\delta \\
= & \ell t^{u_{k-1}}-\ell t^{u_{k-1}}+\ell t^{u_{k}}+\ell_{0}\left(2\left(t^{0}+t^{u_{1}}+\cdots+t^{u_{k}}\right)+\right. \\
& \left.t^{u_{k+1}}+t^{u_{k+1}}+t^{u_{k+2}}+t\right)+\ell-\delta \\
= & f_{k}(t)+\ell\left(t^{u_{k}}-t^{u_{k-1}}\right)+\ell_{0}\left(t^{u_{k+1}}+t^{u_{k+2}}\right) \\
= & f_{k}(t)+g_{k}(t) .
\end{aligned}
$$

Using hypothesis $2 \ell_{0}+\ell-\delta<0$, and (2.28), we get: $f_{0}(0)=2 \ell_{0}+\ell-\delta<0, f_{k}(0)=\delta-\ell<0$ $(k \geq 1)$. Moreover for sufficiently large $t$, we also have $f_{k}(t)>0$ for all $k \geq 0$. It then follows from the intermediate value theorem that: for each $k \geq 0$, there exists $v_{k} \geq 0$, with $f_{k}\left(v_{k}\right)=0$. Each $v_{k}$ is the unique positive zero of $f_{k}$, since $f_{k}^{\prime}(t)>0(k \geq 0)$. That is the graph of function $f_{k}$ crosses the positive axis only once.

By the definition of $f_{k}$ and $v_{k}$, there exists a polynomial $p_{k-1}$ of degree $k-1$, with $p_{k-1}(s)>0$ $(s>0)$, such that:

$$
f_{k}(t)=\left(t-v_{k}\right) p_{k-1}(t) .
$$

To show (2.14), we must have $f_{k}(t) \leq 0$. That is

$$
q=t \leq v_{k} \quad(k \geq 0)
$$

In view of (2.28) and (2.31), we get

$$
\left.f_{k+1}\left(v_{k}\right)=f_{k}\left(v_{k}\right)+g_{k}\left(v_{k}\right)=g_{k}\left(v_{k}\right)=\bar{q}_{k}\left(v_{k}\right) v_{k}^{u_{k-1}} \geq 0 \quad \text { for } \quad v_{k} \geq \delta_{1} \quad \text { (by }(2.27)\right) .
$$

Therefore, if $v_{k} \geq \delta_{1}$, then $v_{k+1} \leq v_{k}$, and $\lim _{k \longrightarrow \infty} v_{k}=q_{\infty}$ exists. Note that $v_{1} \geq \delta_{1}$ by $(2.23)$. Then, it follows $v_{2} \leq v_{1}$. Assume $v_{m} \geq \delta_{1}(m \leq n)$, then $v_{m+1} \leq v_{m}$. We must also show $v_{m+1} \geq \delta_{1}$. But this is true, since $v_{m+1} \geq \delta_{\infty} \geq \delta_{1}$ by (2.23). Then, estimate (2.32) certainly holds if $\delta \leq \delta_{\infty}$, which is true by the choice of $\delta$.

That completes the proof of Lemma 2.2.

We shall study the Secant method (1.2) for triplets $\left(F, x_{-1}, x_{0}\right)$ belonging to the class $\mathcal{C}\left(\ell, \ell_{0}, \eta, c, \delta\right)$ defined as follows:

Definition 2.3. Let $\ell, \ell_{0}, \eta, c, \delta$ be non-negative parameters satisfying the hypotheses of Lemma 2.2.

We say that a triplet $\left(F, x_{-1}, x_{0}\right)$ belongs to the class $\mathcal{C}\left(\ell, \ell_{0}, \eta, c, \delta\right)$ if: 
$\left(c_{1}\right) F$ is a nonlinear operator defined on a convex subset $\mathcal{D}$ of a Banach space $\mathcal{X}$ with values in a Banach space $\mathcal{Y}$;

$\left(c_{2}\right) x_{-1}$ and $x_{0}$ are two points belonging to the interior $\mathcal{D}^{0}$ of $\mathcal{D}$ and satisfying the inequality

$$
\left\|x_{0}-x_{-1}\right\| \leq c
$$

(c $\left.c_{3}\right) F$ is Fréchet-differentiable on $\mathcal{D}^{0}$, and there exists an operator $\delta F: \mathcal{D}^{0} \times \mathcal{D}^{0} \rightarrow \mathcal{L}(\mathcal{X}, \mathcal{Y})$ such that:

the linear operator $A=\delta F\left(x_{-1}, x_{0}\right)$ is invertible, its inverse $A^{-1}$ is bounded and:

$$
\begin{aligned}
\left\|A^{-1} F\left(x_{0}\right)\right\| & \leq \eta \\
\left\|A\left[\delta F(x, y)-F^{\prime}(z)\right]\right\| & \leq \ell(\|x-z\|+\|y-z\|) ; \\
\left\|A\left[\delta F(x, y)-F^{\prime}\left(x_{0}\right)\right]\right\| & \leq \ell_{0}\left(\left\|x-x_{0}\right\|+\left\|y-x_{0}\right\|\right)
\end{aligned}
$$

for all $x, y, z \in \mathcal{D}$.

$\left(c_{4}\right)$ the set $\mathcal{D}_{c}=\{x \in \mathcal{D} ; F$ is continuous at $x\}$ contains the closed ball $\bar{U}\left(x_{0}, t^{\star}\right)=\{x \in X \mid \|$ $\left.x-x_{0} \| \leq t^{\star}\right\}$ where $t^{\star}$ is given in Lemma 2.1.

We present the following semilocal convergence theorem for Secant method (1.2).

Theorem 2.4. If $\left(F, x_{-1}, x_{0}\right) \in \mathcal{C}\left(\ell, \ell_{0}, \eta, c, \delta\right)$, then sequence $\left\{x_{n}\right\}(n \geq-1)$ generated by Secant method (1.2) is well defined, remains in $\bar{U}\left(x_{0}, t^{\star}\right)$ for all $n \geq 0$ and converges to a unique solution $x^{\star} \in \bar{U}\left(x_{0}, t^{\star}\right)$ of equation $F(x)=0$.

Moreover the following estimates hold for all $n \geq 0$

$$
\left\|x_{n+2}-x_{n+1}\right\| \leq t_{n+2}-t_{n+1},
$$

and

$$
\left\|x_{n}-x^{\star}\right\| \leq t^{\star}-t_{n}
$$

where the sequence $\left\{t_{n}\right\}(n \geq 0)$ given by (2.7).

Furthermore, if

$$
\begin{gathered}
\bar{U}\left(x_{0}, \frac{1}{2}\left(c+\frac{1}{\ell_{0}}\right)\right) \subseteq \mathcal{D}, \\
t^{\star \star}<\frac{1}{2}\left(c+\frac{1}{\ell_{0}}\right)
\end{gathered}
$$

the solution $x^{\star}$ is unique in $\bar{U}\left(x_{0}, t^{\star}\right)$.

Finally, if

$$
t^{\star \star}<\frac{1}{\ell_{0}}-R, \quad U\left(x_{0}, R\right) \subseteq \mathcal{D},
$$

where $t^{\star \star}$ is given by (2.8), then the solution $x^{\star}$ is unique in $U\left(x_{0}, R\right)$. 
Proof. We first show operator $L=\delta F\left(x_{k}, x_{k+1}\right)$ is invertible for $x_{k}, x_{k+1} \in \bar{U}\left(x_{0}, t^{\star}\right)$. It follows from $(2.7),(2.8),\left(c_{2}\right)$ and $\left(c_{3}\right)$ that:

$$
\begin{aligned}
\left\|I-A^{-1} L\right\|=\left\|A^{-1}(L-A)\right\| & \leq\left\|A^{-1}\left(L-F^{\prime}\left(x_{0}\right)\right)\right\|+\left\|A^{-1}\left(F^{\prime}\left(x_{0}\right)-A\right)\right\| \\
& \leq \ell_{0}\left(\left\|x_{k}-x_{0}\right\|+\left\|x_{k+1}-x_{0}\right\|+\left\|x_{0}-x_{-1}\right\|\right) \\
& \leq \ell_{0}\left(t_{k}-t_{0}+t_{k+1}-t_{0}+c\right) \\
& \leq \ell_{0}\left(t^{\star}-t_{0}+t^{\star}-t_{0}+c\right) \\
& \leq \ell_{0}\left(2\left[\frac{\eta}{1-\delta}+c\right]-c\right) \leq 1
\end{aligned}
$$

since $\delta \leq \delta_{\infty}$.

According to the Banach Lemma on invertible operators [5], [13], and (2.36), $L$ is invertible and

$$
\left\|L^{-1} A\right\| \leq\left(1-\ell_{0}\left(\left\|x_{k}-x_{0}\right\|+\left\|x_{k+1}-x_{0}\right\|+c\right)\right)^{-1} .
$$

The second condition in $\left(c_{3}\right)$ implies the Lipschitz condition for $F^{\prime}$

$$
\left\|A^{-1}\left(F^{\prime}(u)-F^{\prime}(v)\right)\right\| \leq 2 \ell\|u-v\|, \quad u, v \in \mathcal{D}^{0} .
$$

By the identity,

$$
F(x)-F(y)=\int_{0}^{1} F^{\prime}(y+t(x-y)) d t(x-y)
$$

we get

$$
\left\|A_{0}^{-1}\left[F(x)-F(y)-F^{\prime}(u)(x-y)\right]\right\| \leq \ell(\|x-u\|+\|y-u\|)\|x-y\|
$$

and

$$
\left\|A_{0}^{-1}[F(x)-F(y)-\delta F(u, v)(x-y)]\right\| \leq \ell(\|x-v\|+\|y-v\|+\|u-v\|)\|x-y\|
$$

for all $x, y, u, v \in \mathcal{D}^{0}$. By a continuity argument (2.38)-(2.41) remain valid if $x$ and/or $y$ belong to $\mathcal{D}_{c}$.

We first show (2.33). If (2.33) holds for all $n \leq k$ and if $\left\{x_{n}\right\}(n \geq 0)$ is well defined for $n=0,1,2, \cdots, k$ then

$$
\left\|x_{0}-x_{n}\right\| \leq t_{n}-t_{0}<t^{\star}-t_{0}, \quad n \leq k .
$$

That is (1.2) is well defined for $n=k+1$. For $n=-1$, and $n=0,(2.33)$ reduces to $\left\|x_{-1}-x_{0}\right\| \leq c$, and $\left\|x_{0}-x_{1}\right\| \leq \eta$. Suppose (2.33) holds for $n=-1,0,1, \cdots, k(k \geq 0)$. Using (2.37), (2.41) and

$$
F\left(x_{k+1}\right)=F\left(x_{k+1}\right)-F\left(x_{k}\right)-\delta F\left(x_{k-1}, x_{k}\right)\left(x_{k+1}-x_{k}\right)
$$


we obtain in turn

$$
\begin{aligned}
\left\|x_{k+2}-x_{k+1}\right\| & =\left\|\delta F\left(x_{k}, x_{k+1}\right)^{-1} F\left(x_{k+1}\right)\right\| \\
& \leq\left\|\delta F\left(x_{k}, x_{k+1}\right)^{-1} A\right\|\left\|A^{-1} F\left(x_{k+1}\right)\right\| \\
& \leq \frac{\ell\left(\left\|x_{k+1}-x_{k}\right\|+\left\|x_{k}-x_{k-1}\right\|\right)}{1-\ell_{0}\left[\left\|x_{k+1}-x_{0}\right\|+\left\|x_{k}-x_{0}\right\|+c\right]}\left\|x_{k+1}-x_{k}\right\| \\
& \leq \frac{\ell\left(t_{k+1}-t_{k}+t_{k}-t_{k-1}\right)}{1-\ell_{0}\left[t_{k+1}-t_{0}+t_{k}-t_{0}+t_{0}-t_{-1}\right]}\left(t_{k+1}-t_{k}\right) \\
& =t_{k+2}-t_{k+1} .
\end{aligned}
$$

The induction for (2.33) is now complete. It follows from (2.33) and Lemma 2.2 that sequence $\left\{x_{n}\right\}(n \geq-1)$ is Cauchy in a Banach space $\mathcal{X}$, and as such it converges to some $x^{\star} \in \bar{U}\left(x_{0}, t^{\star}\right)$ (since $\bar{U}\left(x_{0}, t^{\star}\right)$ is a closed set). By letting $k \rightarrow \infty$ in $(2.44)$, we obtain $F\left(x^{\star}\right)=0$.

Estimate (2.34) follows from (2.33) by using standard majoration techniques [1], [5], [13].

We shall first show uniqueness in $\bar{U}\left(x_{0}, t^{\star}\right)$. Let $y^{\star} \in \bar{U}\left(x_{0}, t^{\star}\right)$ be a solution of equation (1.1). Set

$$
\mathcal{M}=\int_{0}^{1} F^{\prime}\left(y^{\star}+t\left(y^{\star}-x^{\star}\right)\right) d t
$$

It then by $\left(c_{3}\right)$ :

$$
\begin{aligned}
\left\|A^{-1}(A-\mathcal{M})\right\| & \leq \ell_{0}\left(\left\|y^{\star}-x_{0}\right\|+\left\|x^{\star}-x_{0}\right\|+\left\|x_{0}-x_{-1}\right\|\right) \\
& \leq \ell_{0}\left(2\left(t^{\star}-t_{0}\right)+t_{0}\right) \\
& \leq \ell_{0}\left(2 t^{\star \star}-c\right)<1
\end{aligned}
$$

since $\delta \leq \delta_{\infty}$.

It follows from (2.35), and the Banach lemma on invertible operators that $\mathcal{M}^{-1}$ exists on $\bar{U}\left(x_{0}, t^{\star}\right)$.

Using the identity:

$$
F\left(x^{\star}\right)-F\left(y^{\star}\right)=\mathcal{M}\left(x^{\star}-y^{\star}\right)
$$

we deduce $x^{\star}=y^{\star}$.

Finally, we shall show uniqueness in $\bar{U}\left(x_{0}, R\right)$. As in (2.45), we arrive at

$$
\left\|A^{-1}(A-\mathcal{M})\right\|<\ell_{0}\left(t^{\star \star}+R\right) \leq 1 .
$$

Hence, again we conclude $x^{\star}=y^{\star}$.

That completes the proof of Theorem 2.4. 
Remark 2.5. Returning back to the example given in the introduction, say $\ell_{0}=.1$, we obtain $\delta_{0}=2.047714554, \frac{1}{c}=5.405$. Condition (2.4) is true, since

$$
\ell_{0}(c+\eta)=.0365<1 \text {. }
$$

Choose $\delta=2.5 \in\left(\delta, \frac{1}{c}\right)$ to obtain $q=.4625$. Then condition (2.6) becomes

$$
.423156212<q \text {. }
$$

That is our results can apply, whereas the ones using (1.3) cannot.

Remark 2.6. Let us define the majoring sequence $\left\{w_{n}\right\}$ used in [4], [5] (under condition (1.3)):

$$
w_{-1}=0, w_{0}=c, w_{1}=c+\eta, w_{n+2}=w_{n+1}+\frac{\ell\left(w_{n+1}-w_{n-1}\right)\left(w_{n+1}-t_{n}\right)}{1-\ell\left(w_{n+1}-w_{0}+w_{n}\right)} .
$$

Note that in general

$$
\ell_{0} \leq \ell
$$

holds, and $\frac{\ell}{\ell_{0}}$ can be arbitrarily large [3], [5]. In the case $\ell_{0}=\ell$, then $t_{n}=w_{n}(n \geq-1)$. Otherwise

$$
\begin{gathered}
t_{n+1}-t_{n} \leq w_{n+1}-w_{n}, \\
0 \leq t^{\star}-t_{n} \leq w^{\star}-w_{n}, \quad w^{\star}=\lim _{n \longrightarrow \infty} w_{n} .
\end{gathered}
$$

Note also that strict inequality holds in (2.49) for $n \geq 1$, if $\ell_{0}<\ell$.

The proof of (2.49), (2.50) can be found in [5]. Note that the only difference in the proofs is that the conditions of Lemma 2.2 are used here, instead of the ones in [4]. However this makes no difference between in the proofs.

We complete this study with an example example to show that $\ell_{0}<\ell$.

Example 2.7. Let $\mathcal{X}=\mathcal{Y}=\mathcal{C}[0,1]$ be the space of real-valued continuous functions defined on the interval $[0,1]$ with norm

$$
\|x\|=\max _{0 \leq s \leq 1}|x(s)| .
$$

Let $\theta \in[0,1]$ be a given parameter. Consider the "Cubic" integral equation

$$
u(s)=u^{3}(s)+\lambda u(s) \int_{0}^{1} q(s, t) u(t) d t+y(s)-\theta .
$$

Here the kernel $q(s, t)$ is a continuous function of two variables defined on $[0,1] \times[0,1]$; the parameter $\lambda$ is a real number called the "albedo" for scattering; $y(s)$ is a given continuous function 
defined on $[0,1]$ and $x(s)$ is the unknown function sought in $\mathcal{C}[0,1]$. Equations of the form (2.51) arise of gasses [5], [8]. For simplicity, we choose $u_{0}(s)=y(s)=1$, and $q(s, t)=\frac{s}{s+t}$, for all $s \in[0,1]$, and $t \in[0,1]$, with $s+t \neq 0$. If we let $\mathcal{D}=U\left(u_{0}, 1-\theta\right)$, and define the operator $F$ on $\mathcal{D}$ by

$$
F(x)(s)=x^{3}(s)+\lambda x(s) \int_{0}^{1} q(s, t) x(t) d t+y(s)-\theta,
$$

for all $s \in[0,1]$, then every zero of $F$ satisfies equation (2.51). We define the divided difference $\delta F(x, y)$ by

$$
\delta F(x, y)=\int_{0}^{1} F^{\prime}(y+t(x-y)) d t
$$

We have the estimate

$$
\max _{0 \leq s \leq 1}\left|\int \frac{s}{s+t} d t\right|=\ln 2 .
$$

Therfore, if we set $b=\left\|F^{\prime}\left(u_{0}\right)^{-1}\right\|$, then, we obtain:

$$
\begin{gathered}
\eta=b(|\lambda| \ln 2+1-\theta) \\
\ell=b(|\lambda| \ln 2+3(2-\theta)) \quad \text { and } \quad \ell_{0}=\frac{1}{2} b(2|\lambda| \ln 2+3(3-\theta)) .
\end{gathered}
$$

Note that $\ell_{0}<\ell$ for all $\theta \in[0,1]$.

Received: October, 2008. Revised: January, 2009.

\section{References}

[1] Argrros, I.K., The theory and application of abstract polynomial equations, St.Lucie/CRC/ Lewis Publ. Mathematics series, 1998, Boca Raton, Florida, U.S.A.

[2] Argyros, I.K., On the Newton-Kantorovich hypothesis for solving equations, J. Comput. Appl. Math., 169 (2004), 315-332.

[3] Argyros, I.K., A unifying local-semilocal convergence analysis and applications for two-point Newton-like methods in Banach space, J. Math. Anal. Appl., 298 (2004), 374-397.

[4] Argyros, I.K., New sufficient convergence conditions for the Secant method, Chechoslovak Math. J., 55, (2005), 175-187.

[5] Argyros, I.K., Convergence and applications of Newton-type iterations, Springer-Verlag Publ., New-York, 2008.

[6] Argyros, I.K. and Hilout, S., Efficient methods for solving equations and variational inequalities, Polimetrica Publ. Co., Milano, Italy, 2009. 
[7] Bosarge, W.E. and Falb, P.L., A multipoint method of third order, J. Optimiz. Th. Appl., 4 (1969), 156-166.

[8] Chandrasekhar, S., Radiative transfer, Dover Publ., New-York, 1960.

[9] Dennis, J.E., Toward a unified convergence theory for Newton-like methods, in Nonlinear Functional Analysis and Applications (L.B. Rall, ed.), Academic Press, New York, (1971), $425-472$.

[10] Gutiérrez, J.M., A new semilocal convergence theorem for Newton's method, J. Comput. Appl. Math., 79 (1997), 131-145.

[11] Hernández, M.A., Rubio, M.J. and Ezquerro, J.A., Secant-like methods for solving nonlinear integral equations of the Hammerstein type, J. Comput. Appl. Math., 115 (2000), $245-254$.

[12] Huang, Z., A note of Kantorovich theorem for Newton iteration, J. Comput. Appl. Math., 47 (1993), 211-217.

[13] Kantorovich and L.V., Akilov, G.P., Functional Analysis, Pergamon Press, Oxford, 1982.

[14] LaAsonen, P., Ein überquadratisch konvergenter iterativer algorithmus, Ann. Acad. Sci. Fenn. Ser I, 450 (1969), 1-10.

[15] Ortega, J.M. And Rheinboldt, W.C., Iterative Solution of Nonlinear Equations in Several Variables, Academic Press, New York, 1970.

[16] Potra, F.A., Sharp error bounds for a class of Newton-like methods, Libertas Mathematica, 5 (1985), 71-84.

[17] Schmidt, J.W., Untere Fehlerschranken fur Regula-Falsi Verhafren, Period. Hungar., 9 (1978), 241-247.

[18] Yamamoto, T., A convergence theorem for Newton-like methods in Banach spaces, Numer. Math., 51 (1987), 545-557.

[19] Wolfe, M.A., Extended iterative methods for the solution of operator equations, Numer. Math., 31 (1978), 153-174. 